\title{
Investigation of Quality Assessment for Different Grades of Velvet Antler: a Strategy Combined Microscopic Observation, Delayed Luminescence and Chemical Analysis
}

\section{Shasha Fan}

Changchun University of Chinese Medicine

Jianxun Zhu

Changchun University of Chinese Medicine

Min He

Changchun University of Chinese Medicine

Nan Wang

Changchun University of Chinese Medicine

Xiaoru Xu

Changchun University of Chinese Medicine

Jingxiang Pang

Shandong Academy of Medical Sciences

Yu Yan

Meluna Research, Koppelsedijk 1-a, 4191LC,Geldermalsen, The Netherlands

Li Li

Capital Medical University subsidiary Beijing Hospital of Traditional Chinese Medicine, No. 23

Backstreet of Art Gallery, Dongcheng District, Beijing, 100010, China

Jiale Yang

Pharmacy Department of University Hospital, Nanhu Campus, Jilin University, No. 5372 Nanhu Rd, ChaoyangDistrict, 130012, Changchun, China

\section{Wen-Te Chang}

College of Chinese Medicine, China Medical University, 91, Hsueh-Shih Road, Taichung, 40402, Taiwan

Tung-Ti Chang

College of Chinese Medicine, China Medical University, 91, Hsueh-Shih Road, Taichung, 40402, Taiwan Jinxiang Han

Shandong Academy of Medical Sciences, NO. 18877 Jingshi Rd, Lixia District, 250062, Jinan, China

Mengmeng Sun ( $\square$ sunm2000@hotmail.com )

SKL of Quality Research in Chinese Medicine, Institute of Chinese MedicalSciences, University of Macau, N22 Avenida da Universidade, Taipa, Macau. 2Changchun University of Chinese Medicine, No. 1035, Boshuo Rd, Jingyue Economic Development District, 130117, Changchun, China 
Research

Keywords: Velvet antler, Delayed luminescence, Protein and amino acids, Microscopic observation, Quality assessment

Posted Date: August 11th, 2020

DOI: https://doi.org/10.21203/rs.3.rs-55925/v1

License: (c) (i) This work is licensed under a Creative Commons Attribution 4.0 International License. Read Full License 


\section{Abstract \\ Background}

As a famous animal-derived herbal medicine, the pharmacological functions of velvet antler are widely recognized in the world. However, there are still great limitations on quality standard and control system of velvet antler.

\section{Methods}

In this research, microscopic observation, delayed luminescence (DL) and chemical analysis were used to evaluate the four grades of velvet antler slices obtained from Cervus nippon Temminck and Cervus elaphus Linnaeu. In addition, those three measurement platforms have been used to compared the two of the four grades among Cervus nippon Temminck, Cervus elaphus Linnaeu and Rangifer tarandus.

\section{Results}

We assessed the quality grades of velvet antler slices by using microscopic observation, delayed luminescence and chemical analysis. These techniques are able to indicate the difference of different grades of velvet antler slices to a certain extent, and has their own advantages, respectively.

\section{Conclusion}

As a new, rapid and systemic tool, DL provides a technique for studying the overall property of different grades of velvet antler slices, and the integrated assessment by measuring microscopic observation, DL and chemical components may provide a novel means to measure animal-derived herbal quality control.

\section{Background}

Velvet antler is a famous traditional Chinese medicine from animal origin that was first recorded in the Shen Nong materia medica written more than 2,000 years ago in China [1]. According to the principles of traditional Chinese medicine, velvet antler is an animal-derived herbal medicine with many therapeutic properties, including the effects of anti-cardiovascular diseases, anti-osteoarthritis and anti-aging etc., to treat a wide range of health problems (Fig. 1) [2, 3]. Therefore, velvet antler is often used as the raw material for various products including herbal drugs and dietary supplements, and the products are popular in many countries, especially in East Asia, North America and New Zealand [4]. In traditional Chinese medicine, velvet antler refers to the immature horn of male deer with thick hair, and without ossification completely [5]. The completely ossified antlers are different from velvet antlers in therapeutic function [6]. The velvet antlers of Cervus nippon Temminck and Cervus elaphus Linnaeu are officially included in the Chinese Pharmacopoeia (2020 Edition) [6]. In the market, the quality grades of velvet 
antler are usually divided into four categories based on the position of the velvet antler slices. The order of the four categories is "LaPian (LP)", "FenPian (FP)", "XuePian (XP)" and "GuPian (GP)" according to the distance from the top of velvet antler (Fig. 1) [7]. This four classifications of velvet antler slices conform to the agricultural industry standard of China (NY/T 1162-2006). LP is located on the top of velvet antler; the ossification level of LP was the lowest compared to the other velvet antler slices. LP is considered to have the best quality and therapeutic function [8], and the price of LP is very expensive. Conversely, the ossification level of GP is relatively high, and the market value is lowest. The common decoction slices of velvet antler are FP and XP, but XP is sometimes impersonated by GP in the market [7]. Given the large annual output of velvet antler in Chinese mainland (approximately 400 tons) and the wide therapeutic application, the quality assessment of velvet antler is very important. However, there are few studies on the quality assessment of animal-derived herbal medicine compared with plant-derived herbal drugs. Chinese Pharmacopoeia (2020 Edition) provides microscopic identification method and thin-layer chromatography method using glycine as the detection index for the quality evaluation of velvet antler [6]. But there is almost no regulation and evaluation standard on the different quality grades of velvet antler. Therefore, to develop a novel quality assessment tool and strategy will help to improve the quality control of velvet antler products.

Delayed luminescence $(\mathrm{DL})$ is the ultra-weak emission of optical photons (wavelength: $400-800 \mathrm{~nm}$ ) from various materials if they were illuminated with excitation light source [9]. DL intensity is radically lower than the well-known fluorescence or phosphorescence, but the decay time of DL is relatively longer (from milliseconds or seconds) [10]. Recently, DL has been used to assess the quality features of dry powders prepared from plant-derived Chinese herbal materials [10-17]. DL provides a new method for measuring plant-derived herbal materials which can be recognized as a rapid, direct and sensitive indicator of a wide range of herbs prepared in different grown environments, different age, different processing statues, as well as different therapeutic properties [11-17]. Thus, DL becomes a promising technique for herbal quality control, and it may become a novel tool to combine with other analytic technological platform such as high performance liquid chromatography fingerprint platform [17]. In order to evaluate whether DL can be used to create a quality assessment of animal-derived herbal medicine. In this study, we performed DL measurements in the dry powders of different grades of velvet antler slices. In addition, microscopic observation and content analysis of protein and amino acid were used to evaluate the quality of the same velvet antler samples. Our results show a high similarity of evaluation effects among those three platforms to differentiate LP, FP, XP and GP in the velvet antler slices of Cervus nippon Temminck and Cervus elaphus Linnaeu, respectively. Moreover, it has the potential to distinguish the non-authentic velvet antler slices from Rangifer tarandus by using DL properties. Thus DL providing a new measurement tool for assessing quality control in velvet antler, even DL has a big potential to be used in the quality evaluation of more animal-derived herbal drugs.

\section{Materials And Methods}

\subsection{Velvet Antler Materials}


The velvet antler samples (LP, FP, XP and GP slices) of Cervus nippon Temminck, Cervus elaphus Linnaeu and Rangifer tarandus were purchased in herbal medicine markets located in Jilin province, China. All samples were verified by Mr. Jianxun Zhu and deposited at Changchun University of Chinese Medicine, Changchun, China. All the velvet antler samples were crushed using a model QE-100 grinder (Yili Company, Zhejiang Province, China), and $150-\mu \mathrm{m}$ and $300-\mu \mathrm{m}$ particles were selected using a standard sieve, respectively. The samples were saved in a dark box containing 3-5-mm silica gel at room temperature.

\subsection{Microscopic Observation}

Microscopic observation is a common method for the identification of Chinese herbal materials [18]. In this study, the powdered velvet antler samples (300- $\mu \mathrm{m}$ particles) were sealed with dilute glycerin when observed under the video capture electron microscope (Model XSZ-N107CCD, Shanghai Guangmi Instrument Co., Ltd., China). The powder of each sample was observed for at least 10 slides. The distinctive representative characteristics under normal light and polarized light microscope were chosen and imaged for observing morphology of the samples.

\subsection{Measurements}

The powdered velvet antler samples (150- $\mu \mathrm{m}$ particles) were stored in a dark box containing 3-5-mm silica gel for $\geq 16 \mathrm{~h}$ before DL detection [10]. The platform for measuring DL (Meluna Research, the Netherlands) included a dark sample chamber with a model 9558QB photomultiplier tube (Electron Tubes Enterprises Ltd., Ruislip, UK). The chamber was kept at $22^{\circ} \mathrm{C}$. The cathode end of the photomultiplier tube has a diameter of $51 \mathrm{~mm}$ and is sensitive at $300-800 \mathrm{~nm}$. The tube was cooled to $-25^{\circ} \mathrm{C}$ to ensure the dark count rate to the level of 10 counts per second. The signal of DL photon emission was amplified using fast preamplifier (model 9301; ORTEC, Oak Ridge, TN). A personal computer containing counting card (model 6602; National Instruments, Austin, TX) was used for signal data acquirement. The velvet antler powder was used to prepare 1-g sample. Each 1-g sample was placed in a Petri dish with 1-cm diameter and excited for $10 \mathrm{~s}$ using a white light emitting diode source. The DL of each sample was measured three consecutive times. The total number obtained from all three measurements in each sample was used to analyze the DL properties of that particular velvet antler sample. DL kinetics were obtained by recording the number of counts in consecutive 0.05 -s periods for a total of 30 -s, resulting in a total of 600 data points.

\subsection{Analysis of Total Protein and Amino Acid Content 2.4.1 Total Protein Content Analysis}

The 2-g velvet antler samples (300- $\mu \mathrm{m}$ particles) were weighted, and placed into a tube. Then $0.4-\mathrm{g}$ of copper sulfate, $6-\mathrm{g}$ of potassium sulphate and $20-\mathrm{mL}$ of sulfuric acid were added into the tube for digestion. When the temperature of the tube reaches $420^{\circ} \mathrm{C}$, the digesting reaction were continued for $1-\mathrm{h}$ until the liquid in the tube is in green color. Thereafter, the liquid was cooled down, and the $50-\mathrm{mL}$ of water was added into the liquid for Kjeldahl analysis. Next, the mixed liquid was injected into the Kjeldahl 
nitrogen analyzer (Model K1100, Hanon Instruments Co., Ltd., Jinan, China), which was added sodium hydroxide solution, sulfuric acid standard solution and boric acid solution containing mixed indicator before use, to test the contents of total protein of velvet antler samples automatically.

\subsubsection{Amino Acid Content Analysis}

An automatic amino acid analyzer (Model L-8900; Hitachi Co., Osaka, Japan) equipped with a visible light detector was used for amino acid content analysis of velvet antler samples (2-g, 300- $\mu \mathrm{m}$ particles). Analytical $2622(4.6 \times 60 \mathrm{~mm})$ and guard $2650(4.6 \times 40 \mathrm{~mm})$ columns were used for the determination of 16 amino acids. Immediately after injection of the sample into the columns, an auto-sampler was used for in-line derivatization by NIN post-column derivatization. NIN-derivatized proline was detected at $440 \mathrm{~nm}$ and the other amino acids were detected at $570 \mathrm{~nm}$. The contents of 16 amino acids were expressed automatically by amino acid analyzer.

\subsection{Data Processing and Statistical Analysis 2.5.1 Statistics of Chemical Data}

The contents of total protein in LP, FP, XP and GP slices were calculated for statistical analysis. Subsequently, a two-tailed, unpaired Student's t-test was performed (SPSS version 23.0; IBM, Armonk, NY) to compare the different grades of velvet antler slices; differences were considered significant at $p<0.05$. The heat-map analysis was performed using the data of amino acid contents to compare the differences of 16 amino acids among LP, FP, XP and GP of the velvet antler slices.

\subsubsection{Statistics of DL Data}

The DL photon counts were used to calculate the properties by using hyperbolic function [] in order to obtain four DL parameters (i.e., I0, Beta, Tau, T). The three measurements were averaged and used to represent the DL properties of each velvet antler sample. Principal component analysis (PCA) was used to show the level of discrimination among DL properties of LP, FP, XP and GP slices. A two-tailed, unpaired Student's t-test was used (SPSS version 23.0) to compare the different grades of velvet antler slices; differences were considered significant at $p<0.05$. In addition, Spearman's rank correlation $(\rho)$ was used to quantify the correlation between the 16 identified amino acids and four DL properties. Strong linear relationship was defined as Spearman's $|\rho|>0.70$ [19]. Thereafter, Cytoscape version 3.2.1 (www.cytoscape.org) was used to draw a network view [20], which was used visualize these correlations.

\section{Results}

To evaluate the quality of velvet antler samples, microscopic observation, DL measurements and chemical analysis were used to assess the LP, FP, XP and GP slices obtained from Cervus nippon Temminck and Cervus elaphus Linnaeu, respectively. We first focused on the experimental results of Cervus nippon Temminck. Figure 2 illustrates the images of LP, FP, XP and GP powder of Cervus nippon Temminck., and the selected slides of hair, bone fragments and non-ossified bone tissue. It was observed 
that the hair of LP samples was relatively smooth, and the bone density of LP was relatively higher from the image of bone fragments. Compared to LP, the hair of FP, XP and GP was relatively rough (FP), less $(\mathrm{XP})$ and thin (GP), respectively; the bone fragments of FP, XP and LP possessed more bone lacunas; and the non-ossified bone tissue of FP, XP and LP demonstrated more irregular massive protuberances. Next, the LP, FP, XP and GP samples was measured by using DL. Figure 3a illustrates the significantly different DL decay curves of LP, FP, XP and GP powder. To extract the four parameters, a hyperbolic function was used to fit the $D L$ decay curves. To analyze further the difference between DL parameters, a two-tailed, unpaired Student's t-test was used to compare the four DL parameters among the LP, FP, XP and GP samples. The results showed that the parameter I0 was significantly different in all velvet antler samples; T, Beta and Tau differed significantly among LP, FP and XP samples, but these parameters were not able to indicate the significant difference between XP and GP powder. To further evaluate the DL data, PCA was applied to the DL data for visualizing the variations among $d$ LP, FP, XP and GP samples. The results illustrated that there were significant classifications among those samples (Fig. 3c). Many research supports that main prominent bioactive components of velvet antler are proteins and polypeptides [21]. Therefore, we tested the contents of total protein and 16 amino acids to assess the quality of LP, FP, XP and GP samples. The results showed that the total protein contents decreased gradually and significantly from LP to GP (Fig. 3d). Figure 3e is a heat-map of the tested amino acids in LP, FP, XP and GP samples. The contents of most amino acids in LP samples were significantly high, and the contents of most amino acids in GP samples were significantly low. LP, FP, XP and GP samples contained specific amino acids with characteristics in contents, respectively. For instance, the contents of Arg and Leu were highest in LP; the contents of Pro and Val were relatively lowest in FP; Tyr, Ala showed highest contents in XP; and Pro and Lys showed relatively highest and lowest contents, respectively, in GP. Next, we pooled DL and amino acids data of LP, FP, XP and GP samples together to establish a network in order to indicate the correlation between DL and specific chemicals in the velvet antler slices of Cervus nippon Temminck. The DL parameters possessed positive and strong correlation with Phe, Met, Glu, Asp and Arg (Fig. 3f).

Next, we focused on the experimental results of Cervus elaphus Linnaeu. In the results of microscopic observation, we found that the LP powder possessed more hair fragments compared to the FP, XP and GP powder. The hair fragments of FP powder were rough and thick, and the hair fragments of XP and GP powder were relatively thin (Fig. 4). It is worth noting that the morphology of bone fragments and nonossified bone tissue of FP, XP and GP powder were different compared that of Cervus nippon Temminck. There were no significant bone lacunas and irregular massive protuberances, but darker colors on bone fragments and non-ossified bone tissue of XP powder and more fragments of bone and non-ossified bone tissue of GP powder (Fig. 4). In DL measurements results, the decay curves of LP, FP, XP and GP slices demonstrated differences, but the tails of curves overlapped together (Fig. 5a). The parameter I0 was able to distinguish LP, FP, XP and GP powder, but the other DL parameters were not able to indicate the significantly difference among them completely (Fig. 5b). In the PCA analysis, there was no clear separation between FP and LP samples, but XP and GP samples showed significantly different classification compared with LP and FP samples (Fig. 5c). The results of total protein contents also showed a gradual and significant decrease from LP to GP slices (Fig. 5d). LP samples demonstrated the 
most amino acids with high contents, however, GP samples also contained a lot of high contents amino acids compared to FP and XP slices (Fig. 5e). In additional, some characteristic amino acids were found such as Ser, Leu and Arg (LP); Gly and His (FP); Ala and Lys (XP); and Pro and Val (GP) (Fig. 5e). Moreover, the correlation network showed that there were nine positive and strong correlations between amino acids and DL data (Fig. 5d).

Next, the velvet antler samples of Rangifer tarandus were examined in order to test whether microscopic observation, DL measurements and chemical analysis could distinguish the samples which are not officially included in Chinese Pharmacopoeia. This is because of a lot of non-official velvet antler products exist on the market. Here, FP and XP samples obtained from Rangifer tarandus were used for this study. In the results of microscopic observation (Fig. 6), the hair fragments of FP and XP samples showed no significant difference compared to that of Cervus nippon Temminck and Cervus elaphus Linnaeu. The FP powder of Rangifer tarandus had no significant bone lacunas and irregular massive protuberances, but more non-ossified bone tissues. The XP powder of Rangifer tarandus had irregular massive protuberances on non-ossified bone tissue. Next, the DL decay curves of FP slices showed significant different photon emission kinetics among the three species of deer (Fig. 7a). Subsequently, all the DL parameters demonstrated significant difference of FP slices among those three species of deer (Fig. 7b). However, the DL decay kinetics of XP slices showed relatively minor difference (Fig. 7c), and no single DL parameter of XP slices was able to separate the three species of deer completely (Fig. $7 \mathrm{~d}$ ). In the analysis of chemical data, there was no significant difference on total protein contents of FP samples between Cervus nippon Temminck and Cervus elaphus Linnaeu, but the FP samples of Rangifer tarandus showed significantly lower contents of total protein compared to that of Cervus nippon Temminck and Cervus elaphus Linnaeu (Fig. 7e). In XP samples, there was no significant difference of total protein contents among those three species of deer (Fig. 7e). In addition, the results of heat-map illustrated that the contents of specific amino acids were significant difference on FP and XP samples, respectively, among the three species of deer (Fig. $7 \mathrm{f}$ and $7 \mathrm{~g}$ ).

\section{Discussion}

As a famous animal-derived herbal drugs, the pharmacological functions of velvet antler are widely recognized in the world. However, there are still great limitations on quality standard and control system of velvet antler in China. The traditional identification methods of velvet antler are morphological observation based on the appearance, shape, size and color of velvet antler samples [22]. However, it is difficult to identify velvet antler samples by experience-based observation method if the velvet antler samples are prepared to powder or processed products [5]. In addition, there are different quality grades of velvet antler slices in the market, it is difficult to ensure the accuracy and stability of identification only by morphological observation, even the research on quality assessment of different grades of velvet antler slices is still limited. Therefore, we have used microscopic observation and chemical analysis, which has been recommended to evaluate velvet antler quality in Chinese pharmacopoeia, as well as a systemic and rapid DL measurement to exanimate the different slices (i.e. LP, FP, XP and GP) of velvet antler among the three different species of deer. 
In this research, using microscopic observation method could partly distinguish LP, FP, XP and GP samples based on the observation index (i.e., hair, bone fragments and non-ossified bone tissue) regulated in Chinese pharmacopoeia. However, well-trained and experienced experts are still needed to determine the differences among velvet antler samples, which leads to a probability of longer identification time and lower efficiency. In addition, the microscopic identification method could not reveal the composition of velvet antler. Therefore, we conducted chemical analysis to further investigate the contents differences of total protein and amino acids among velvet antler samples with different grades. Velvet antlers are rich in proteins, amino acids and polypeptides which are considered as the most prominent bioactive components, and the contents of these components are more than half percentage in velvet antler [23]. Therefore, the examination of crude protein contents based on Kjeldahl method may reflect the quality of velvet antler samples with different grades. In our research, the content of total protein decreased significantly in turn from LP to GP, which supported the market grades of velvet antler slices. In Chinese Pharmacopoeia (2020 Edition), Gly is the only chemical index to indicate the quality of velvet antler. In the heat-map results, the contents of Gly in LP, FP, XP and GP samples demonstrated a certain degree of gradient differences in Cervus nippon Temminck and Cervus elaphus Linnaeu, respectively, as well as the comparison among FP and XP samples of the three species of deer. The results showed that the existing evaluation index (Gly) of Chinese Pharmacopoeia may be extended to characterize the quality grades of velvet antler slices. In addition, the contents of most amino acids in LP samples was highest, which supports again the LP samples of velvet antler may have better quality. Moreover, we found several characteristic amino acids among LP, FP, XP and GP samples, these amino acids were able to indicate the difference among velvet antler slices with different quality grades or different species of deer. The results reveal that the profile of the multiple characteristic amino acids may show better identification effect. In fact, some studies [24, 25] have used metabolomics methods to analyze different grades of velvet antler slices, and suggest integrated evaluation index to distinguish velvet antler slices by using more metabolites including amino acids, fatty acids and organic acids etc. Now, the research show that polypeptides may be the most important bioactive markers of velvet antler [26]. However, the highly specific polypeptide which can be used as a quality marker of velvet antler has not been separated effectively. Therefore, it is a feasible method to evaluate the quality grades of velvet antler from the profile of various chemical components at present.

DL is a new technique to evaluate the quality of Chinese herbal medicine. In this study, we first applied DL to assess the quality of animal-derived herbal drugs. We found DL decay curves and parameters demonstrated strong identification effect to distinguish LP, FP, XP and GP samples of Cervus nippon Temminck, as well as FP samples among the three species of deer. But The identification effect of DL to distinguish different graded slices of Cervus elaphus Linnaeu was slightly weak, as well as XP samples among the three species of deer. In general, DL shows a good evaluation effect on velvet antler samples in this study. Many studies have proved that the variability of DL is closely related to the chemical structure of samples, especially long-chain macromolecules such as polysaccharides [27]. This is because of the changes of the conformation and structure of sample's molecule can affect the kinetics of luminescence [28]. Polypeptides and proteins are typical long-chain bio-macromolecules, we found LP 
and FP samples of Cervus nippon Temminck possessed stronger DL decay kinetics and parameters, and the contents of total protein of LP and FP samples are also much higher than that of XP and GP samples. However, although the total protein content of XP samples was significantly higher than that of GP samples, there was no significant difference in most DL parameters (Beta, Tau and T, Fig. 3b) between XP and GP of Cervus nippon Temminck. This is because of the content of protein cannot reflect the specific structures of protein molecules, and the different molecule structures of protein are main factors to affect DL emission [29,30]. So, the different protein contents of velvet antler slices could not correspond the different DL properties in the samples of Cervus nippon Temminck completely, as well as the comparison of XP and GP slices among those three species of deer. In addition, the strong and positive correlations have been found between DL parameters and amino acids. Most of these amino acids shows significant difference among LP, FP, XP and GP slices in the heat-map analysis. Therefore, DL parameters may be used as objective markers to reflect the grades differences of velvet antler slices. In brief, DL is a comprehensive and systemic measurement, reflecting the overall chemical information of the samples. In the absence of specific chemical quality markers, it may be a feasible method to identify velvet antler products by using DL. Recently, spectral technology, such as ultraviolet and near-infrared technology, has been used for the quality control study of velvet antler products [5]. The wavelength range of DL is different from these two technologies, likely to provide new information for the quality assessment of velvet antler, and DL measurements may be further benefit for the quality control of other animal-derived herbal medicine.

\section{Conclusion}

In this research, we assessed the quality grades of velvet antler slices by using microscopic observation, delayed luminescence and chemical analysis. These techniques are able to indicate the difference of different grades of velvet antler slices to a certain extent, and has their own advantages, respectively. As a rapid detection technology, DL shows great potential for evaluating velvet antler products. It indicates that $\mathrm{DL}$ is a promising method to evaluate animal-derived herbal medicine. At present, more than 50 kinds of animal-derived herbal medicine and more than 400 kinds of Chinese patent medicines containing animal-derived drugs are included in the Chinese Pharmacopoeia. However, only more than ten kinds of animal-derived drugs can be determined the exclusive active ingredients for their quality assessment [31] Therefore, all the methods that can be used to evaluate animal-derived drugs need to be further explored. This proof-of-concept study may provide a suitable foundation for follow-up studies. And the further research should be repeated using a wider range of velvet antler samples. In conclusion, DL provides a technique for studying the overall property of different grades of velvet antler slices, and the integrated assessment by measuring microscopic observation, DL and chemical components may provide a novel means to measure herbal quality control.

\section{Abbreviations}

$\mathrm{DL}$ 
Delayed luminescence; PCA:Principal component analysis; Alanine:Ala; Valine:Val; Leucine:Leu; Isoleucine:Ile; Fhenylalanine:Phe; Methionine:Met; Proline:Pro; Glycine:Gly; Tyrosine:Tyr; Serine:Ser; Threonine:Thr; Asparticacid:Asp; Glutamicacid:Glu; Lysine:Lys; Histidine:His; Arginine:Arg.

\section{Declarations}

\section{Ethics approval and consent to participate}

Not applicable.

\section{Consent for publication}

The manuscript is approved by all authors for publication.

\section{Availability of data and material}

The datasets used in this study are available from the corresponding author upon reasonable request.

\section{Competing interests}

All contributing authors declare no conflicts of interest.

\section{Funding}

This research was supported by "Macao Young Scholars Program (No. AM201928)".

\section{Authors' contributions}

SF, JZ, and MS designed the study. MS, JP, YY and JH conducted the DL measurements and statistical analyses. JZ and XX carried out microscopic observation and data analysis. LL and NW performed chemical analysis and data analysis. SF drafted the manuscript and $\mathrm{MH}$ prepared tables and figures. WTC, TTC and JY contributed to revisions of the manuscript. All authors read and approved the final manuscript.

\section{Acknowledgments}

This research was supported by "Macao Young Scholars Program (No. AM201928)". The authors thank the support from Dr. Eduard Van Wijk and Meluna Research, the Netherlands.

\section{Authors' information}

Corresponding authors:

Mengmeng Sun: sunm2000@hotmail.com; Jinxiang Han: samshjx@sina.com

E-mail addresses of the other authors: 
530983236@qq.com (Shasha Fan); 347450519@qq.com (Jianxun Zhu); heminccucm@hotmail.com (Min He); 169610026@qq.com (Nan Wang); emilyxuxiaoru@163.com (Xiaoru Xu); sdamspjx@163.com (Jingxiang Pang); yan@melunaresearch.nl (Yu Yan); lyx9981@163.com (Li Li); wtchang@mail.cmu.edu.tw (Wen-Te Chang); tchang0604@yahoo.com.tw (Tung-Ti Chang); yangjiale@jlu.edu.cn (Jiale Yang)

\section{References}

1. Sun Q. Research on the anti-aging medicines of ancient Chinese Materia Medica books. China Academy of Chinese Medical Sciences. Doctoral Dissertation. 2017.

2. Sui Z, Zhang L, Huo Y, Zhang Y. Bioactive components of velvet antlers and their pharmacological properties. J Pharmaceut Biomed. 2014;87:229-40. http://dx.doi.org/10.1016/j.jpba.2013.07.044.

3. Zhang L, Wang J, Li T, Li P, Wang Y, Yang M, Liu J, Liu JY. Determination of the chemical components and phospholipids of velvet antler using UPLC/QTOF-MS coupled with UNIFI software. Exp Ther Med. 2019;17:3789-99. https://doi.org/10.3892/etm.2019.7372.

4. Lee S, Yang H, Ding Y, Wang Y, Jeon Y, Moon S, Jeon B, Sung S. Anti-inflammatory effects of enzymatic hydrolysates of velvet antler in RAW 264.7 cells in vitro and zebrafish model. Excli J. 2015;14:1122-32. DOI:10.17179/excli2015-481.

5. Luo W, Zhao H, Qi X, Guan Q, Liu J, Zhang Z, Li C. Research progress on identification and quality control of Cervi Cornu Pantotrichum. China J Chin Mater Med. 2017;42(21):4110-4. DOI:10.19540/j.cnki.cjcmm.20171016.002.

6. Pharmacopoeia of the People's Republic of China. Beijing, China Medical Science Press; 2020.

7. Liu X. Quality analysis of different specifications of antler pieces. Changchun University of Chinese Medicine. Master Dissertation. 2019.

8. Gong W, Li F. Quality evaluation on different specifications of Cervi Cornu Pantotrichum with its effect on ovariectomized osteoporosis model rats. China J Chin Mater Med. 2014;39(12):2326-9. DOI:10.4268 /cjcmm20141235.

9. Scordino A, Baran I, Gulino M, Ganea C, Grasso R, Niggli J, Musumeci F. Ultraweak delayed luminescence in cancer research: a review of the results by the ARETUSA equipment. J Photochem Photobiol B. 2014;5:76-84. https://doi.org/10.1016/j.jphotobiol.2014.03.027.

10. Sun M, Van Wijk R, Van Wijk E, Wang M, Wietmarschen H, Hankemeier T, van der Greef J. Delayed luminescence: an experimental protocol for Chinese herbal medicines. Luminescence. 2016;31(6):1220-8. DOI:10.1002/bio.3094.

11. Sun M, Chang WT, van Wijk E, He M, Koval S, Lin MK, van Wijk R, Hankemeier T, van Greef J, Wang M. Characterization of the therapeutic properties of Chinese herbal materials by measuring delayed luminescence and dendritic cell-based immunomodulatory response. Photochem Photobiol B Biol. 2017;168:1-11. http://dx.doi.org/10.1016/j.jphotobiol.2017.01.018. 
12. Sun M, Li L, Wang M, van Wijk E, He M, van Wijk R, Koval R, Hankemeier T, van Greef J, Wei S. Efects of growth altitude on chemical constituents and delayed luminescence properties in medicinal rhubarb. Photochem Photobiol B Biol. 2016;162:24-33.

http://dx.doi.org/10.1016/j.jphotobiol.2016.06.018.

13. Sun M, Chang WT, Van Wijk E, He M, Van Wijk R, Wang M. Application of delayed luminescence method on measuring of the processing of Chinese herbal materials. Chin Med. 2018;13(1):43. https://doi.org/10.1186/s13020-018-0202-0.

14. Sun M, Wang S, Jing Y, Li L, He M, Jia Y, Van Wijk E, Wang Y, Wang Z, Wang M. Application of delayed luminescence measurements for the identifcation of herbal materials: a step toward rapid quality control. Chin Med. 2019;14(1):47. https://doi.org/10.1186/s13020-019-0269-2.

15. Sun M, He M, Korthout H, Halima M, Kim HY, Yan Y, Van Wijk E, Van Wijk R, Guo C, Wang M. Characterization of ginsenoside extracts by delayed luminescence, high-performance liquid chromatography, and bioactivity tests. Photochem Photobiol Sci. 2019;18:1138-46. DOI:10.1039/c8pp00533h.

16. Jia Y, Sun M, Shi Y, Zhu Z, Van Wijk Eduard, Van W, Roeland, van Andel Tinde, Wang M. A comparative study of aged and contemporary Chinese herbal materials by using delayed luminescence technique. Chin Med. 2020;15:6. https://doi.org/10.1186/s13020-020-0287-0.

17. Sun M, Wu H, He M, Jia Y, Wang L, Liu T, Hui L, Li L, Wei S, Van Wijk E, Van Wijk R, Tsim K, Li C, Wang M. Integrated assessment of medicinal rhubarb by combination of delayed luminescence and HPLC fngerprint with emphasized on bioactivities based quality control. Chin Med. 2020;15:72. https://doi.org/10.1186/s13020-020-00352-8.

18. Xu Y, Song W, Zhou P, Li P, Li H. Morphological and microscopic characterization of fve commonlyused testacean traditional chinese medicines. Acta Pharm Sin B. 2015;5(4):358-66. http://dx.doi.org/10.1016/j.apsb.2015.03.014.

19. He M, Van Wijk E, van Wietmarschen H, Wang M, Sun M, Koval S, Van Wijk R, Hankemeier T, van der Greef J. Spontaneous ultra-weak photon emission in correlation to inflammatory metabolism and oxidative stress in a mouse model of collagen-induced arthritis. Photochem Photobiol Sci. 2017;168:98-106. https://doi.org/10.1016/j.jphotobiol.2016.12.036.

20. Cline M, Smoot M, Cerami E, Kuchinsky A, Landys N, Workman C, Christmas R, Avila-Campilo I, Creech M, Gross B, Hanspers K, Isserlin R, Kelley R, Killcoyne S, Lotia S, Maere S, Morris J, Ono K, Pavlovic V, Pico A, Vailaya A, Wang P, Adler A, Conklin B, Hood L, Kuiper M, Sander C, Schmulevich I, Schwikowski B, Warner G, Ideker T, Bader G. Integration of biological networks and gene expression data using cytoscape. Nat Protoc. 2007;2(10):2366-82. DOI:10.1038/nprot.2007.324.

21. Gong R, Wang Y, Gao K, Zhang L, Liu C, Wang Z, Wang Y, Sun Y. Quantification of Furosine (Ne-(2Furoylmethyl)-I-lysine) in Different Parts of Velvet Antler with Various Processing Methods and Factors Affecting Its Formation. Photochem Molecules. 2019;24(7):1255.

DOI:10.3390/molecules24071255. 
22. Yao M, Hu S, Zhang H, Sun J. Quality evaluation of cartialgenous and its products. Jilin J Tradit Chin Med. 2016;36(6):594-7. DOI: 10.13463 /j.cnki.jlzyy.2016.06.016.

23. Ding Y, Ko S, Moon S, Lee S. Protective Effects of Novel Antioxidant Peptide Purified from Alcalase Hydrolysate of Velvet Antler Against Oxidative Stress in Chang Liver Cells In Vitro and in a Zebrafish Model In Vivo. Int J Mol Sci. 2019;20(20):5187. DOI:10.3390/ijms20205187.

24. Sun W, Zhao H, Wang X, Li G. Analysis on differential metabolites of Cervi Cornu Pantotrichum based on metabolomics. Zhong Cao Yao. 2019;20:5047-53. DOI:10.7501/j.issn.0253-2670.2019.20.029.

25. Liu W, Li C, Ba H, Zhao H. Studies on untargeted metabolomics of the four portions of velvet antlers in Sika deer (Cervus nippon). J Jilin Agr Univ. 2019. DOI:10.13327/j.jjlau.2019.4627.

26. Zhao L, Wang X, Zhang X, Xie Q. Purification and identification of anti-inflammatory peptides derived from simulated gastrointestinal digests of velvet antler protein (Cervus elaphus Linnaeus). J Food Drug Anal. 2016;24:376-84. https://doi.org/10.1016/j.jfda.2015.10.003.

27. Grasso R, Musumeci F, Triglia A, Brizhik L, Scordino A. Impact of structure on the delayed luminescence of d-Glucose-based polymer chains. J Photochem Photobiol B. 2019;1:111589. DOI:10.1016/j.jphotobiol.2019.111589.

28. Barenboïm GM, Domanskii AN, Turoverov KK. Luminescence of biopolymers and cells. New York: Springer; 1969.

29. Ho M, Musumeci F, Scordino A, Triglia A. Privitera G. Delayed luminescence from bovine Achilles' tendon and its dependence on collagen structure. J Photochem Photobiol B. 2002;66:165-70. DOI:10.1016/S1011-1344(02)00239-7.

30. Scordino A, Grasso R, Gulino M, Lanzanò L, Musumeci F, Privitera G, Tedesco M, Triglia A, Brizhik L. Delayed luminescence from collagen as arising from soliton and small polaron states. Int $\mathrm{J}$ Quantum Chem. 2010;110:221-9. D0I:10.1002/qua.22010.

31. Qiu Y, Deng Y, Che J, Ma H, Shen G. Review of Animal Drug Identification. J Chengdu Univ Tradit Chin Med. 2019;42(2):74-7. DOI:10.13593/j.cnki.51-1501/r.2019.02.074.

\section{Figures}




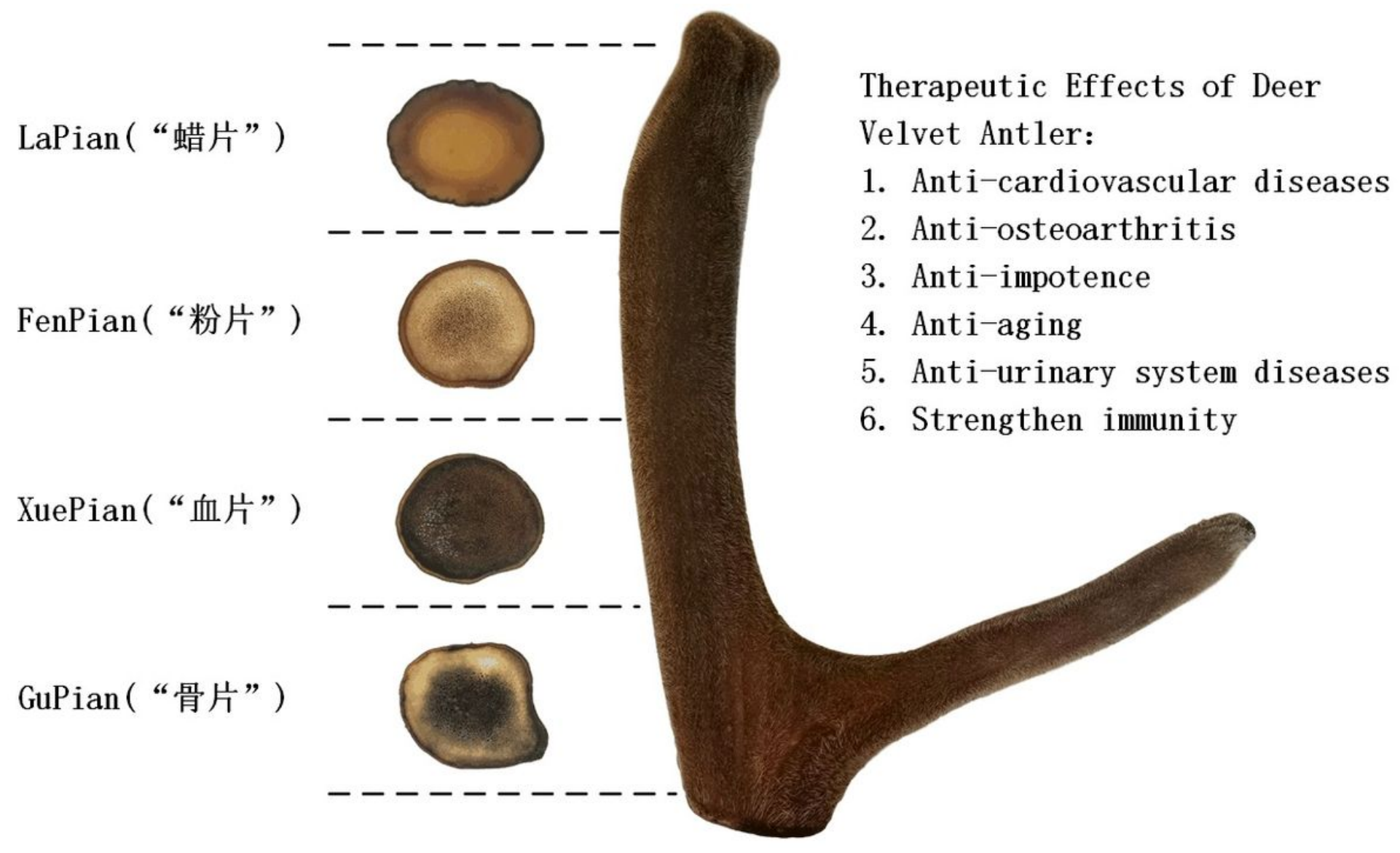

Figure 1

Schematic diagram of the therapeutic effects and quality grades of velvet antler.
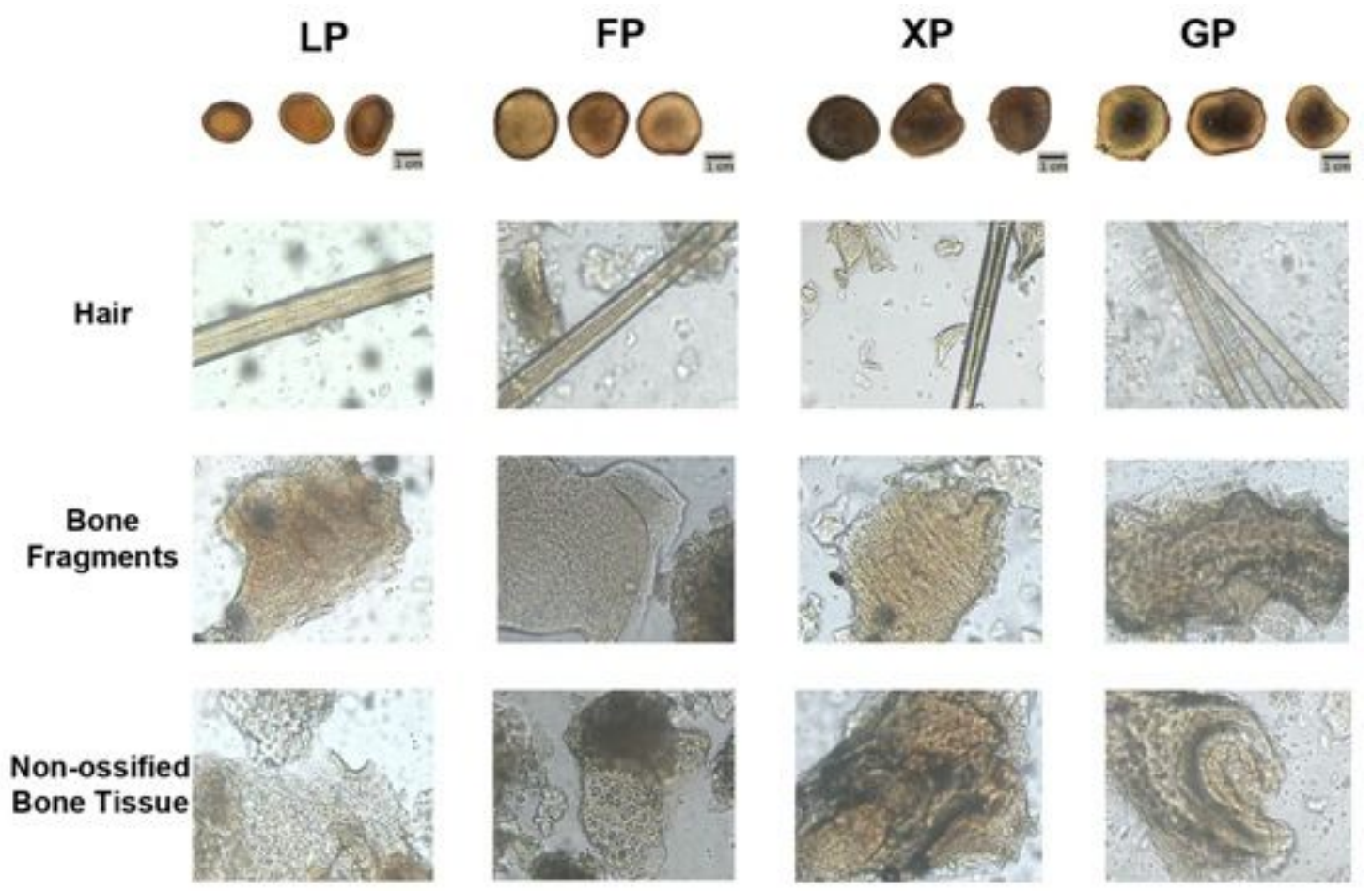

Figure 2 
Microscopic observation of LP, FP, XP and GP samples of velvet antler (Cervus nippon Temminck). Magnification: 160 times.
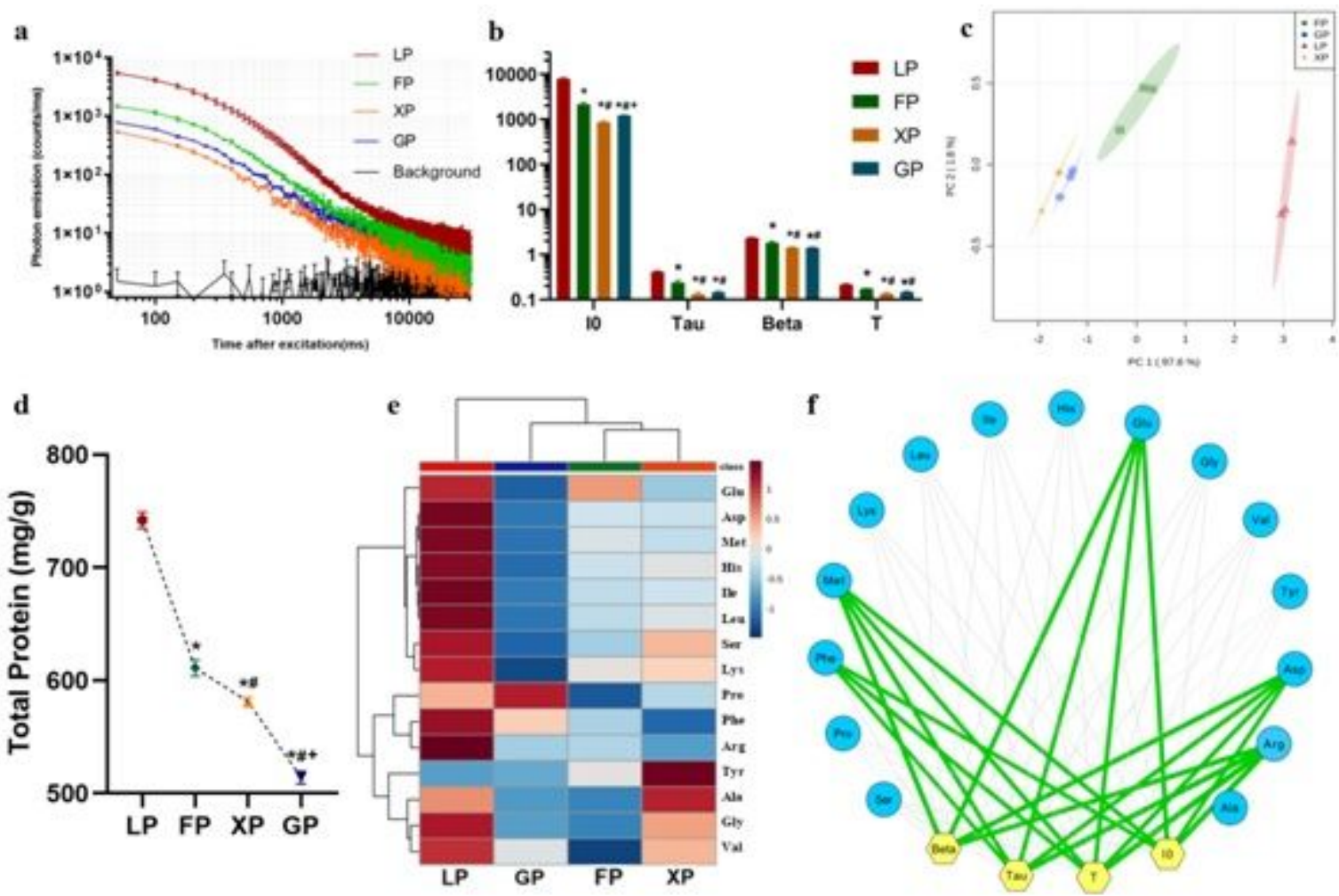

\section{Figure 3}

DL measurements and chemical analysis of the velvet antler samples (Cervus nippon Temminck). a) DL decay curves for LP, FP, XP and GP. Data are plotted as the mean \pm SEM. Note that the data are plotted on a log-log scale; b) Histograms comparing the DL properties among LP, FP, XP and GP. * indicates FP, XP or $G P$ versus $L P, p<0.05$. \# indicates $X P$ or $G P$ versus $F P, p<0.05$. + indicates $X P$ versus $G P, p<0.05$. Beta is an index factor associated with the rate of $D L$ decay, 10 is the initial intensity of the $D L$ curve, and $T$ and Tau represents the decay time and DL characteristics, respectively; c) PCA score plots of the DL properties obtained from all samples of LP, FP, XP and GP; $d$ ) The difference of total protein contents among LP, FP, $X P$ and GP. * indicates FP, XP or GP versus $L P, p<0.05$. \# indicates XP or GP versus $F P, p<0.05 .+$ indicates XP versus GP, $p<0.05$; e) Heat-map of amino acids of LP, FP, XP and GP; $f$ ) Correlation network between the identified amino acids and DL properties measured in the LP, FP, XP and GP samples. The positive and strong correlations are indicated with green lines $(|\rho|>0.70)$. The other correlations $(|\rho|<$ $0.70)$ are indicated with grey lines. Thicker lines indicate a stronger correlation, and the length of each line has no meaning. 


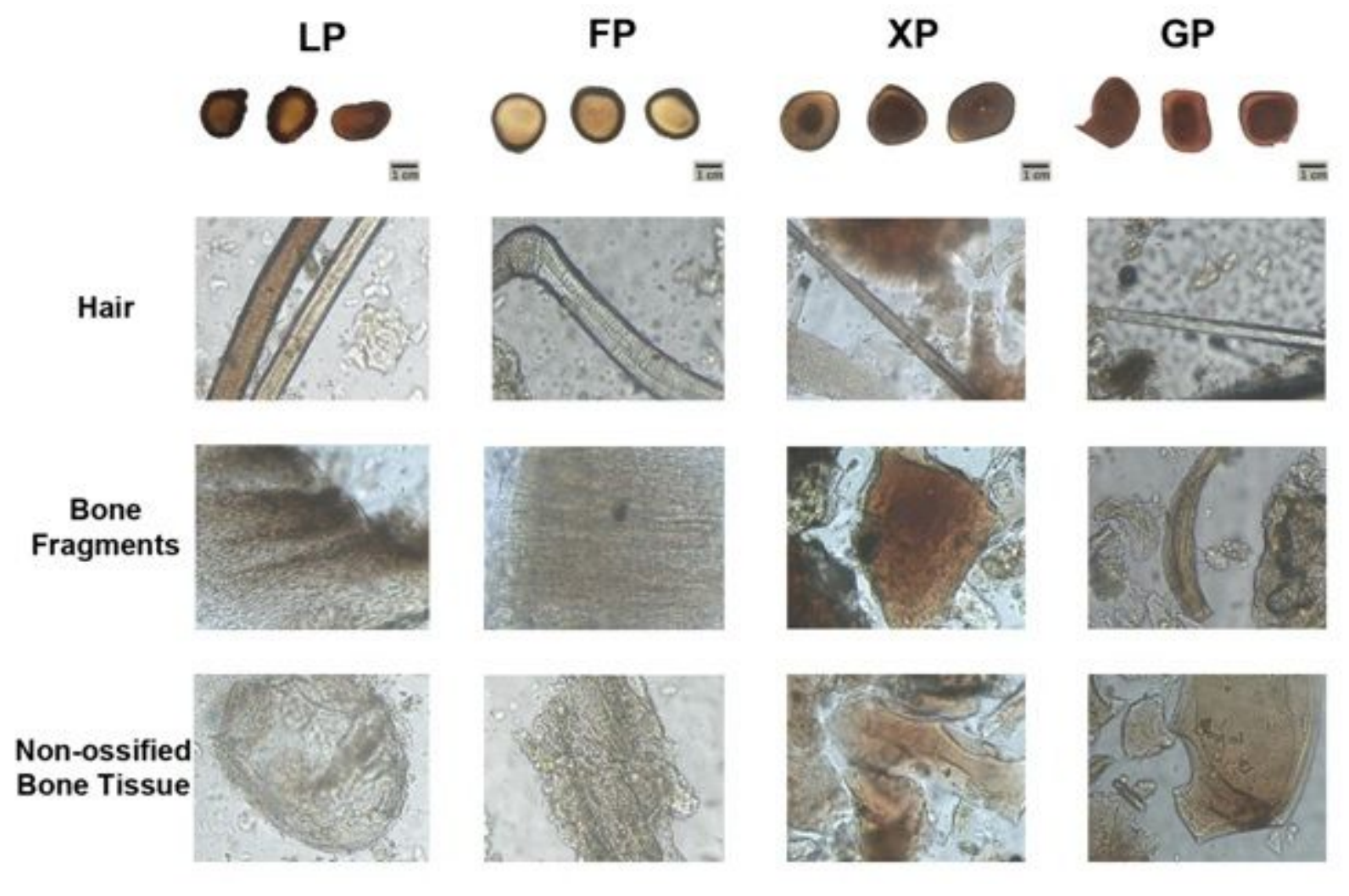

Figure 4

Microscopic observation of LP, FP, XP and GP of velvet antler (Cervus elaphus Linnaeu). Magnification: 160 times.
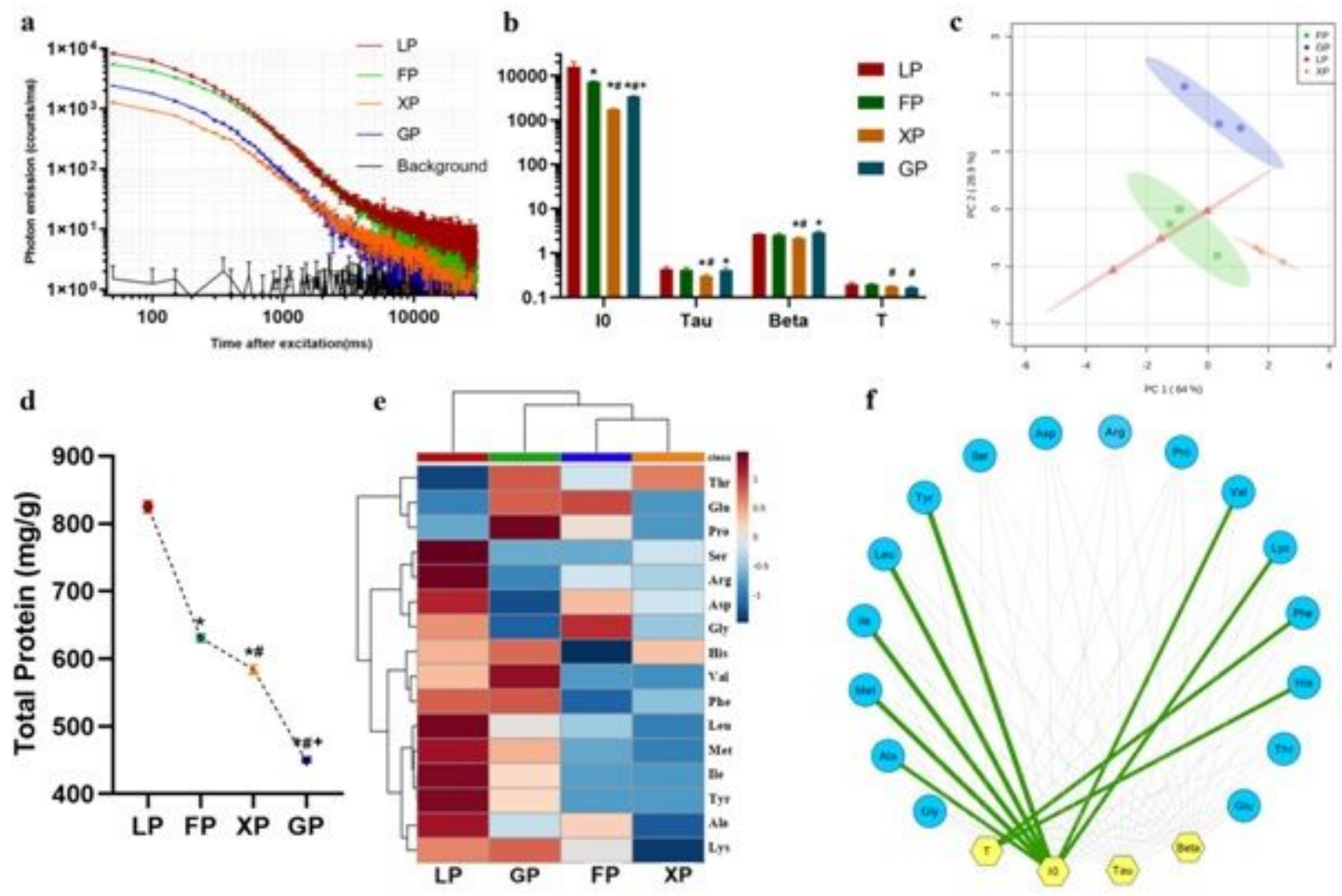

Figure 5 
DL measurements and chemical analysis of the velvet antler samples (Cervus elaphus Linnaeu). a) DL decay curves for LP, FP, XP and GP. Data are plotted as the mean \pm SEM. Note that the data are plotted on a log-log scale; $b$ ) Histograms comparing the DL properties among LP, FP, XP and GP * indicates FP, XP or GP versus $L P, p<0.05$. \# indicates $X P$ or GP versus FP, $p<0.05$. + indicates $X P$ versus $G P, p<0.05$. Beta is an index factor associated with the rate of $D L$ decay, 10 is the initial intensity of the $D L$ curve, and $T$ and Tau represents the decay time and DL characteristics, respectively; c) PCA score plots of the DL properties obtained from all samples of LP, FP, XP and GP; d) The difference of total protein contents among LP, FP, $X P$ and GP. * indicates FP, XP or GP versus $L P, p<0.05$. \# indicates $X P$ or GP versus $F P, p<0.05$. + indicates XP versus GP, $p<0.05$; e) Heat-map of amino acids of LP, FP, XP and GP; f) Correlation network between the identified amino acids and DL properties measured in the LP, FP, XP and GP samples. The positive and strong correlations are indicated with green lines $(|\rho|>0.70)$. The other correlations $(|\rho|<$ $0.70)$ are indicated with grey lines. Thicker lines indicate a stronger correlation, and the length of each line has no meaning.

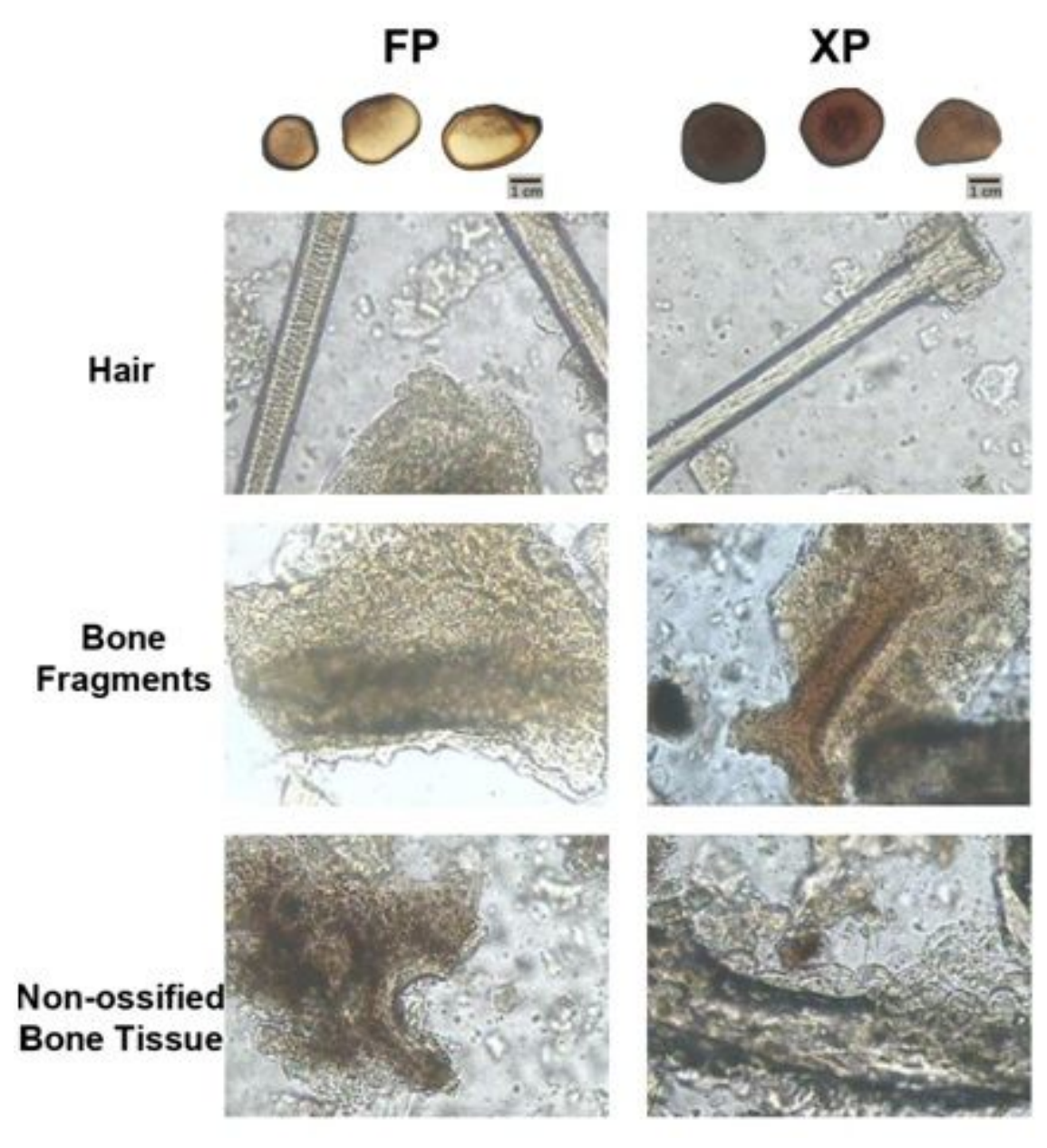

\section{Figure 6}

Microscopic observation of FP and XP of velvet antler (Rangifer tarandus). Magnification: 160 times. 
a

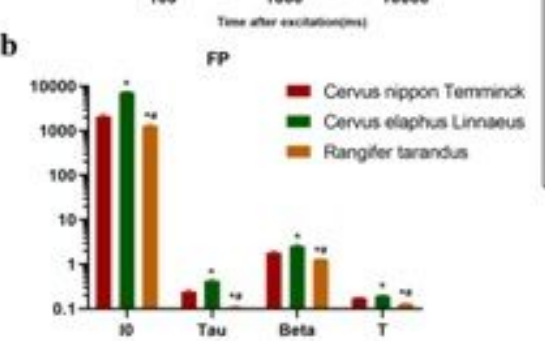

c

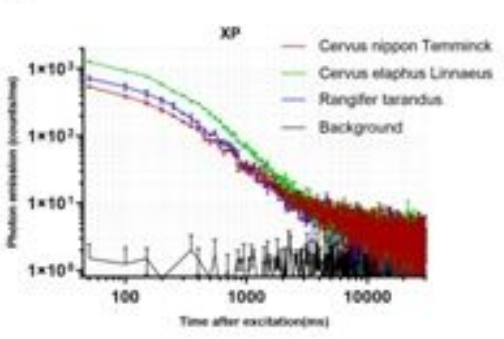

f

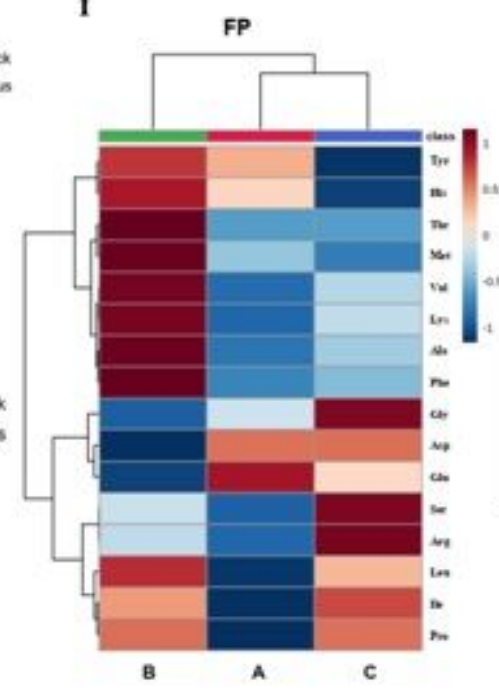

d

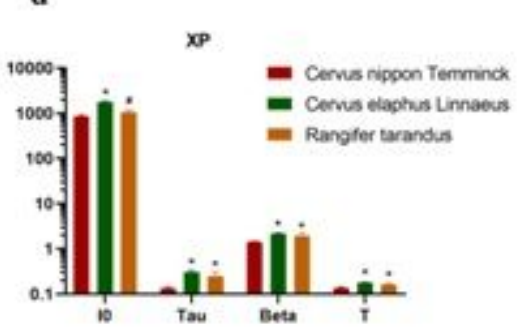

g

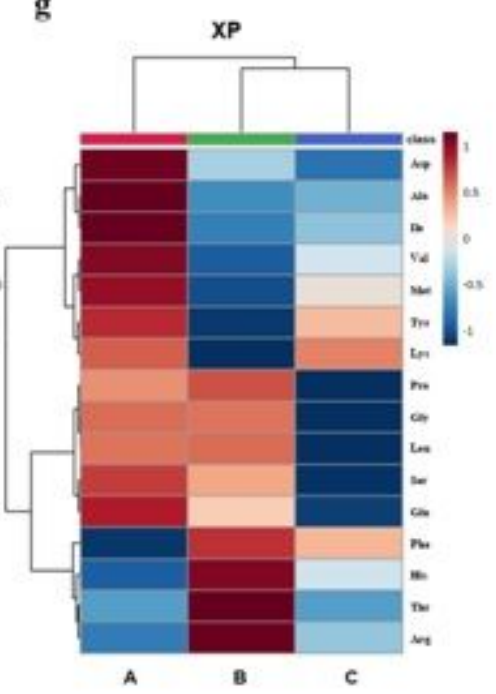

e

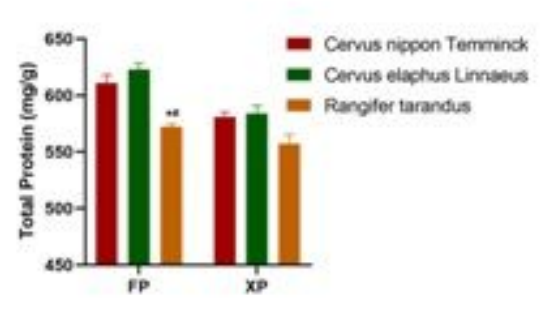

Figure 7

Comparison of DL measurements and chemical analysis of the velvet antler samples from the three species of deer. a) and c) DL decay curves for FP and XP among the velvet antler samples from the three species of deer. Data are plotted as the mean \pm SEM. Note that the data are plotted on a log-log scale; $b$ ) and d) Histograms comparing the DL properties of FP and XP among the velvet antler samples from the three species of deer, respectively. * indicates Cervus elaphus Linnaeu or Rangifer tarandus versus Cervus nippon Temminck, $p<0.05$. \# indicates Cervus elaphus Linnaeu versus Rangifer tarandus, $p<0.05$. Beta is an index factor associated with the rate of DL decay, 10 is the initial intensity of the DL curve, and $T$ and Tau represents the decay time and DL characteristics, respectively; e) Histograms comparing the total protein contents of FP and XP among the velvet antler samples from the three species of deer, respectively. * indicates Cervus elaphus Linnaeu or Rangifer tarandus versus Cervus nippon Temminck, $p$ $<0.05$. \# indicates Cervus elaphus Linnaeu versus Rangifer tarandus, $p<0.05$. f) and g) Heat-map of amino acids of FP and XP among the velvet antler samples from the three species of deer, respectively. " $A$ " indicates the samples from Cervus nippon Temminck. " $B$ " indicates the samples from Cervus elaphus Linnaeu. " $C$ " indicates the samples from Rangifer tarandus. 